Meta

Journal des traducteurs

Translators' Journal

\title{
Traduction, poésie, figement et jeux de mots
}

\section{Salah Mejri}

Volume 45, numéro 3, septembre 2000

La traduction dans le monde arabe

URI : https://id.erudit.org/iderudit/003612ar

DOI : https://doi.org/10.7202/003612ar

Aller au sommaire du numéro

Éditeur(s)

Les Presses de l'Université de Montréal

ISSN

0026-0452 (imprimé)

1492-1421 (numérique)

Découvrir la revue

Citer cet article

Mejri, S. (2000). Traduction, poésie, figement et jeux de mots. Meta, 45(3),

412-423. https://doi.org/10.7202/003612ar

\section{Résumé de l'article}

Le passage de L1 à L2 lors de la traduction de textes poétiques, de séquences figées et de jeux de mots est révélateur de l'existence de plusieurs degrés de déperdition qui, tout en étant très différents - en poésie, il est possible de conserver certains transferts tropiques; dans les séquences figées, seule l'existence d'équivalents rend la traduction possible; dans les jeux de mots, le blocage est presque total - rapprochent ces trois domaines en mettant en relief l'importance de la matérialité du signe linguistique telle qu'elle s'exprime à travers ses spécificités phoniques et ses combinaisons syllabiques et rythmiques.
Ce document est protégé par la loi sur le droit d'auteur. L'utilisation des services d'Érudit (y compris la reproduction) est assujettie à sa politique d'utilisation que vous pouvez consulter en ligne.

https://apropos.erudit.org/fr/usagers/politique-dutilisation/ 


\title{
Traduction, poésie, figement et jeux de mots
}

\author{
SALAH MEJRI \\ Université de Tunis I, Tunis, Tunisie
}

\begin{abstract}
RÉSUMÉ
Le passage de $L_{1}$ à $L_{2}$ lors de la traduction de textes poétiques, de séquences figées et de jeux de mots est révélateur de l'existence de plusieurs degrés de déperdition qui, tout en étant très différents - en poésie, il est possible de conserver certains transferts tropiques; dans les séquences figées, seule l'existence d'équivalents rend la traduction possible; dans les jeux de mots, le blocage est presque total — rapprochent ces trois domaines en mettant en relief l'importance de la matérialité du signe linguistique telle qu'elle s'exprime à travers ses spécificités phoniques et ses combinaisons syllabiques et rythmiques.
\end{abstract}

\section{ABSTRACT}

The change from $L_{1}$ to $L_{2}$ in the translation process of poetic texts, fixed phrases and plays on words reveals the existence of several degrees of loss which, though diverse in poetry it is possible to keep some tropic transfers; in fixed phrases only the existence of equivalents makes translation possible; in plays on words, the blockage is almost complete - contribute to bring the three fields closer by highlighting the importance of the linguistic sign materiality as expressed through phonic specificities as well as syllabic and rythmic combinations.

\section{MOTS-CLÉS/KEYWORDS}

traduction, poésie, figement, jeux de mots, dénomination

Parmi les questions les moins débattues en traduction, nous retenons celles de la poésie, des séquences figées (SF) et des jeux de mots (JM), trois domaines apparemment sans rapports directs mais qui semblent avoir des liens étroits que la traduction met en relief d'une manière empirique. En effet, il suffit de comparer des traductions en rapport avec ces trois domaines pour s'en convaincre.

Bien qu'il s'agisse de trois entités différentes - le figement étant un processus linguistique, la poésie et le JM deux usages particuliers de la langue —, plusieurs aspects les rapprochent.

Nous essaierons dans ce qui suit de montrer en quoi ils se ressemblent, comment la traduction aide à fonder ce rapprochement tout en illustrant notre analyse par des exemples empruntés à divers types de textes.

\section{Constat empirique}

Il est communément admis que les SF résistent à la traduction; il en est de même des JM. Des exemples comme (1) et (2):

(1) - À qui profite le crime? À qui profite l'écrit, à qui profitent les cris? C'est toi qui choisis d'écrire ce que tu décris ou de décrire ce que tu écris. (Constant 1998: 177). - «Double, double, puis redouble, le feu chante au chaudron trouble», répliqua Lola dans un anglais clair [...]. (Constant 1998: 181) 
- [...] Aurore va nous écrire une oraison funèbre: Aurora, or aux rats, Horrora. (Constant 1998: 233) [C'est nous qui soulignons.]

(2) Gervaise s'amusa à suivre trois ouvriers, un grand et deux petits, qui se retournaient tous les dix pas: ils finirent par descendre la rue, ils vinrent droit à l'Assommoir du père Colombe.

- Ah bien! murmura-t-elle, en voilà trois qui ont un fameux poil dans la main. (Zola, L’Assommoir, t. 1, p. 47, cité par Rey 1989) [C'est nous qui soulignons.]

fournissent des cas où la traduction en arabe par exemple détruirait l'effet du jeu ou ne rendrait pas compte des nuances exprimées par la SF:

- En (1), le jeu sur les paronymes ou les homophones employés dans le même cadre syntaxique :

- À qui profite le crime? $\rightarrow$ [manilmustafi:d minalzari:ma] ?

- À qui profite l'écrit? $\rightarrow$ [manilmustafi:d minalmaktu:b] ?

- À qui profitent les cris? $\rightarrow$ [manilmustafi:d minassija:h] ?

la segmentation différenciée du même segment:

- Aurora $\rightarrow$ [orora] (conservation du nom propre)

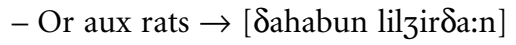

ou l'effet de rime:

- Double, double, puis redouble... trouble. $\rightarrow$ [da: $:$ if $\underline{\text { da }}: \div$ if $\theta$ umma $\underline{\text { da }}: \div$ if $\ldots$ kadir $]$

à l'exception de ce dernier cas où les mêmes termes se répètent, toutes ces spécificités disparaissent purement et simplement.

- En (2), même si le signifié d' "avoir un poil dans la main» (être très paresseux) demeure traduisible, le passage d'une langue à l'autre ne se fait pas sans déperdition.

Ainsi, dans un cas comme dans l'autre, les seules possibilités qui s'offrent au traducteur se réduisent soit à la stricte information soit à une imitation du jeu dans la langue d'arrivée sans qu'il y ait la moindre garantie de réussite dans la transposition.

(1) et (2) comparés à (3) représentent des cas limites où la marge de l'universel est réduite au maximum et où le spécifique — d'aucuns diraient l'idiomatique l'emporte sur les autres dimensions de la langue:

(3) La voiture avance à une très grande vitesse.

Le singe grimpe à l'arbre avec une rapidité étonnante.

$$
\begin{aligned}
& \rightarrow \quad \text { [tatakaddamussajja:ratu } \\
& \text { bisur } \div \text { atin kabi:ratin ziddan]. }
\end{aligned}
$$

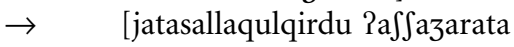

$$
\begin{aligned}
& \text { bisur } \div \text { atin mudhilatin]. }
\end{aligned}
$$

Qu'est-ce qui oppose ces deux types d'exemples?

Il est évident que (3) représente des phrases où syntaxe et lexique concourent pour véhiculer un contenu informationnel strict. Comparés à (1) et (2), ces exemples n’impliquent le lexique que dans ce qu'il a de référentiel et de dénotatif. Cela signifie que les mots, en tant que signes d'autre chose, renvoient par leur présence dans la chaîne syntagmatique aux références correspondantes d'une manière directe, c'est-àdire sans l'interférence d'autres références.

Tel n'est pas le cas des métaphores dans (4) qui, tout en étant traduisibles, se construisent sur la base de l'interposition de la référence des mots utilisés entre ce qui est dit et ce qui est signifié: 
(4) «Les livres d'aujourd'hui sont en papier. Les livres d'hier étaient en peau. La Bible est le seul livre d'air — un déluge d'encre et de vent.» (Bobin 1992: 13)

[inna kutubaljawma min waraqin / ka:nat kutubul?amsimin zildin / wattawra:tu hijja Pawwalu kita:bin min hawa:?in / tu:fa:nun min hibrin warịhin]

Pourquoi une telle interposition ne perturbe-t-elle pas la traduction? Pourquoi ne remet-elle pas en question l'emploi dénotatif et direct des mots?

La traduction de ce passage nous offre l'occasion de voir comment les unités lexicales, tout en connaissant un décrochage référentiel par lequel elles ne désignent plus les entités auxquelles elles renvoient initialement, sont susceptibles des mêmes emplois dans un autre système linguistique, ce qui nous fournit le moyen de réfuter l'idée selon laquelle, si les textes poétiques résistent à la traduction, c'est parce qu'ils sont fondés sur l'enchevêtrement des constructions tropiques, qu'il s'agisse de métaphore ou de métonymie (cf. aussi [5]):

(5) «La chambre était nue et froide, vidée par l'ivrognerie de l'homme qui enlevait les draps du lit pour les boire.» (Zola, cité par Bonhomme 1987)

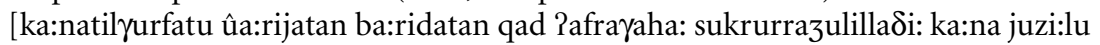
a

Avec la métonymie, au lieu de la suspension référentielle, il y a incompatibilité ontologique (cf. Prandi 1998: 34-44) mais, malgré cela, le passage de $\mathrm{L}_{1}$ à $\mathrm{L}_{2}$ ne pose pas de problèmes particuliers. Même s'il est vrai que ces énoncés, pris à la lettre, donneraient des suites incompréhensibles, il n'en demeure pas moins qu'il est toujours possible de les reproduire dans un système comme dans l'autre.

\section{La déperdition inéluctable dans la traduction poétique}

Si on prend des passages poétiques français et arabe et qu'on leur applique la même opération, on s'apercevra que la traduction demeure possible, ce qui explique et justifie les différentes traductions du même texte, malgré certaines déperditions:

(6a) Cordes faites de cris

$\rightarrow$ [hiba:lun minsura:xin

Sons de cloches à travers l'Europe $\rightarrow$ daqquliazra:si $\div$ abra?u:rubba

Siècles pendus

Rails qui ligotez les nations

Nous ne sommes que deux

ou trois hommes

Libres de tous liens

$\rightarrow$ quru:nun mu $\div$ allaqatun

$\rightarrow$ Rajjatuhassikakullati: tu:Oiqul?umama

$\rightarrow$ nahnu lasn: siwa: razulajni ?aw

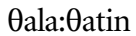

$\rightarrow$ Rahra:run min zami:-:i lquju:di]

(Apollinaire 1925: 23)

(6b) Inonde la brillance de tes yeux L'ensemble de mes mains

$\leftarrow$ [jansa:bu bari:qu $\div$ ajnajki

Et s'établisse dans mes mots

Le chant de tes seins

$\leftarrow$ bajna Pana:mili:

$\leftarrow$ wajastaqirru Sadwu nahdajki

$\leftarrow$ fi:kalima:ti:]

(anonyme)

À partir de ces exemples, il semble que la résistance à la traduction ne se situe ni au niveau de l'information, ni au niveau du décrochage référentiel, ni même au niveau de l'incompatibilité ontologique. Pourquoi de tels mécanismes ne bloquent-ils pas la traduction? Parce que tout simplement, dans le cas de l'information pure, c'est la fonction dénotative propre à toutes les langues qui conditionne les divers emplois 
des signes linguistiques; dans celui de l'incompatibilité ontologique, c'est la sélection sémique opérée au niveau du contexte qui oriente la compréhension.

Il arrive que des écarts dus aux habitudes linguistiques soient réalisés, mais le message demeure transposable d'une langue à l'autre.

Qu'est-ce qui bloque donc la traduction dans les textes poétiques?

Partant des comparaisons que nous venons de faire, il semble évident que le blocage est situé hors des mécanismes sémantiques généraux et des multiples opérations impliquées par les divers transferts que connaît le texte poétique. C'est du côté de la matérialité du signe linguistique qu'il faudrait chercher à déterminer les difficultés à traduire certains faits; nous nous contentons d'en mentionner les suivants:

a. La matérialité du signe linguistique peut être appréhendée à plusieurs niveaux d'analyse. Cela peut concerner les combinaisons phoniques qui constituent la trame sonore des unités lexicales, laquelle joue un rôle déterminant dans les associations lexicales fondées le plus souvent sur la recherche d'effets mélodiques et rythmiques. Or, la structure syllabique, les regroupements consonantiques et les distributions vocaliques sont autant d'éléments spécifiques à chaque langue. Y toucher, c'est engager «l'intimité» de la langue.

b. La configuration morphologique des unités lexicales est un autre niveau d'analyse qui nous permet de voir comment les associations lexicales se font sur la base de critères morphologiques, la morphologie étant conçue comme tout ce qui a trait à la forme des unités lexicales.

Les niveaux phonémique et morphémique conditionnent dans la poésie toutes sortes de constructions rythmiques et décident des alternances des assonances et des allitérations le plus souvent exploitées pour seconder les mécanismes sémantiques ou même s'y substituer en ancrant le sens dans la musicalité des mots, c'est-à-dire dans l'empreinte matérielle de la langue.

c. Le niveau syntaxique, même s'il ne représente pas un élément de blocage à la traduction, impose les particularités de chaque système, ce qui donne des transpositions et des «accommodations» qui peuvent porter atteinte à la poéticité des passages traduits. Dans (6b), la traduction française proposée de vers arabes a respecté, par exemple, l'ordre syntaxique des phrases verbales arabes verbe-sujet-complément, ce qui n'est pas toujours possible.

Ainsi, ce que nous avons appelé matérialité de la langue n'est en fait rien d'autre qu'un ensemble de spécificités phoniques, syllabiques, morphémiques, lexicales et syntaxiques appréhendées en tant que telles, c'est-à-dire dans leur expression et non dans leur fonctionnalité. En d'autres termes, la présence du signe linguistique dans la chaîne parlée suffit à participer au sens par ses sonorités, sa configuration et sa position dans l'énoncé.

C'est ce que la traduction participe à mettre en relief: dans les textes poétiques, on est en mesure de conserver lors du passage de $\mathrm{L}_{1}$ et $\mathrm{L}_{2}$ les correspondants lexicaux, les images créées par les associations lexicales et syntaxiques, une partie des isotopies structurant le texte, mais la déperdition est plus ou moins importante selon qu'on réussit ou non à créer à partir de la matérialité des signes de la langue d'arrivée, ce qui serait l'équivalent des choix réalisés dans la langue-source. C'est la raison pour laquelle on admet plusieurs traductions du même texte (cf. par exemple les multiples traductions en arabe du poème «Le lac» de Lamartine). La différence se situe souvent au niveau de la création poétique dans la langue d'arrivée. En fait, il s'agit beaucoup plus de réécriture que de traduction: aussi paradoxal que cela puisse paraître, 
plus la traduction de la poéticité du texte est réussie, plus la part de réécriture est importante; plus on est fidèle au texte d'origine, c'est-à-dire moins la traduction de la poéticité est réussie, moins la part de la réécriture est importante (cf. Radhouane 2000).

\section{La rupture inévitable dans la traduction des SF}

Si on entend par SF les différents types de syntagmes qui perdent leur syntaxe libre au profit d'un fonctionnement général très contraint, il semble évident que de telles séquences constituent des unités propres à chaque langue qu'il serait très difficile de rendre lors du passage d'une langue à l'autre.

Tel n'est pas notre propos; ce qui nous intéresse, c'est l'analyse linguistique nous permettant de saisir à quel niveau se situe la rupture lors du passage de $\mathrm{L}_{1}$ à $\mathrm{L}_{2}$. Que font les traducteurs devant une telle difficulté? De quels critères devrait-on se servir pour apprécier les solutions qu'ils avancent? Ces questions sont d'autant plus importantes que le nombre de SF semble être aussi important que celui des séquences dites libres (SL) (M. Gross 1982).

Si on reprend les éléments de définition avancés plus haut, il y a lieu d'en dégager les remarques suivantes:

1) Une SF est une unité polylexicale syntaxiquement bien formée et constituant objectivement une sorte de «doublet» avec la SL d'origine qui demeure remotivable à n'importe quel moment. Dire de quelqu'un qu' «il a un poil dans la main » peut très bien signifier "être très paresseux» ou, moyennant quelques éléments contextuels, tout simplement avoir le sens dit compositionnel. Cela donnerait alors lieu à deux traductions différentes:

(7a) [lahu Sa:ratun fi:jadihi] (traduction arabe littérale)

(7b) [innahu kasu:lun ziddan] $\rightarrow$ Il est très paresseux.

2. La dualité structurelle à la fois formelle et sémantique (syntaxe libre/syntaxe figée ; sens compositionnel/sens non compositionnel) engendre nécessairement une rupture entre la SF et la SL; si la SL implique des unités lexicales fonctionnant avec leur potentiel sémantique et référentiel, et par conséquent la liberté syntaxique qui en est le corollaire, il n'en est pas de même de la SF dont l'intégrité sémantique et référentielle des constituants est entamée à un niveau quelconque à l'occasion de manipulations syntaxiques, ce qui donne lieu à une syntaxe contrainte. Dans

(8) Il a cassé sa pipe.

on ne relève pas seulement les deux traductions objectivement possibles:

(8a) [qad kassara yulju:nahu] (traduction littérale)

(8b) [qad ma:ta] $\rightarrow$ Il est mort.

mais le décrochage référentiel des unités lexicales constitutives et les implications sémantiques qui s'ensuivent ont pour conséquence le blocage de certaines transformations syntaxiques — comme la passivation — qui, une fois employées, ne sélectionnent que la traduction littérale:

(8c) Sa pipe a été cassée. $\rightarrow$ [kussira $\gamma u l j u: n u h u]$ 
3. Le plus souvent, le figement, de par cette dualité structurelle, joue le rôle d'un catalyseur culturel, dans ce sens que son intervention a pour conséquence des opérations de transferts sémantiques dont le résultat est une sorte d'ancrage culturel dans la langue, désigné couramment par «les façons de parler»ou «l'idiomaticité ». Dans une situation de traduction, il est indispensable d'en tenir compte soit en trouvant des correspondants aux SF de $\mathrm{L}_{1}$ soit en leur substituant des équivalents. Dans un cas comme dans l'autre, la rupture avec les constituants libres est inéluctable. Dans (9) et (10), nous disposons de deux SF dans lesquelles figure le même élément nominal «veste» qui, lors du passage à $\mathrm{L}_{2}$, en l'occurrence l'arabe littéral, ne peut pas avoir de correspondant, non parce que ce terme n'existe pas en $\mathrm{L}_{2}$ mais parce que son statut de constituant en $\mathrm{L}_{1}$ dans la SF ne lui permet pas un tel transfert:

(9) prendre une veste, 'subir un échec'

(10) retourner sa veste, 'changer brusquement et totalement d'opinion, de parti'

Mais cela ne signifie nullement que les correspondants figés n'existent pas. Pour ces mêmes exemples, sous l'effet probablement des calques, on trouve des séquences similaires en arabe dialectal tunisien:

(9a) [kla:kabbu:t] (littéralement: manger une capote ${ }^{1}$ )

(10a) [qlibilvi:sta] (calque de « retourner sa veste»)

De tels correspondants demeurent toutefois très rares.

La disparition de ces constituants en tant que tels est la règle; l'exception est leur conservation. Un tel fonctionnement s'explique par les particularités de transfert de domaines que le figement réalise dans chaque langue en usant du lexique de certains domaines pour exprimer ou dénommer des réalités appartenant à d'autres domaines. Le résultat, c'est la lexicalisation des désignations partagées par chaque communauté linguistique et, par conséquent, leur fixation dans le lexique. Une fois intégrées dans le lexique, les SF cessent d'être perçues à travers leurs motivations premières. Or, puisqu'une des caractéristiques des SF est d'avoir un signifiant-étymon dont la reconnaissance ne relève pas des compétences du spécialiste mais de celles du locuteur ordinaire, il serait évident que la trace de la première motivation de la SF soit perçue comme une saillance culturelle lors du passage de $L_{1}$ à $L_{2}$. Cette saillance est d'autant plus importante que les langues n'opèrent pas les mêmes transferts de domaines. Comparez à titre d'exemples (11) et (12) formés en français et en arabe avec le mot «nez»:

(11) «au nez de quelqu'un », 'en échappant à sa vigilance'; «à plein nez», 'très fort'; «à vue de nez», 'approximativement'; «le nez au vent», 'en flânant'; "avoir du nez» et «avoir le nez creux», 'avoir du flair'; « avoir quelqu'un dans le nez», 'ne pas le supporter'; «bouffer le nez de (à) quelqu'un », 'l'insulter'; "se casser le nez», 'subir un échec'; etc.

(12) [jatba $\div$ u Ranfahu], littéralement 'suivre son nez' : 'suivre les autres et les espionner'; [?anfu qawmihi], litt. 'le nez de sa communauté' : 'le maître de la communauté'; [jahmi: ?anfahu], litt. 'protéger son nez': 'ne pas supporter l'injustice'; [ $\int a m a x a b i$ ?anfihi], litt. 'élever son nez' : 'avoir l'air hautain'; etc.

Ces exemples nous fournissent la preuve que l'emploi de mots propres à des domaines - ici le nez comme partie du corps - dans l'expression de faits appartenant à d'autres domaines (la proximité, le flair, la poéminence, l'expression d'attitudes diverses, etc.) nécessite une démarche qui tienne compte de la rupture effectuée 
entre le sens compositionnel et les éléments de sens en rapport avec les domaines dénommés par les SF.

Pour rendre compte d'une telle rupture, seuls les équivalents unilexicaux ou paraphrastiques peuvent traduire les SF auxquelles il n'y a pas de correspondants polylexicaux:

(13) bouffer le nez de (à) quelqu'un à vue de nez

$$
\begin{aligned}
& \rightarrow[\text { atamahu }], \text { 'l'insulter' } \\
& \rightarrow[\div \text { an qurbin }], \text { 'tout près' }
\end{aligned}
$$

ce qui consacre la rupture entre ce qui est dit, c'est-à-dire la SF dans la matérialité linguistique que sont les composants de son signifiant, et ce qui est signifié: dans $\mathrm{L}_{2}$, c'est le signe qui véhicule le signifié global de la SF qui sert de substitut lors de la traduction.

Partant de cette remarque, nous pouvons faire le rapprochement avec la poésie dont la traductibilité bute contre les données de base des signifiants investis dans le poème: tout comme pour la poésie où la déperdition consiste en l'abandon des spécificités sonores et rythmiques, dans les SF, c'est le signifiant polylexical — avec sa saillance culturelle, ses connotations sémantiques et, éventuellement, ses remotivations possibles - qui se trouve séparé de son signifié lors de la traduction, un signifié dont le support phonique (= le signifiant) en $\mathrm{L}_{2}$ pourrait être le correspondant unilexical de son synonyme en $\mathrm{L}_{1}$, le correspondant polylexical en $\mathrm{L}_{2}$ ou une paraphrase:

\section{L1}

$\mathrm{SF}$ «casser sa pipe»

$\mathrm{SF}$ « rendre le dernier soupir»

$\mathrm{SF}$ «tourner autour du pot»

\section{L2}

(a) unité unilexicale ([ma:ta], 'mourir')

(b) SF correspondante ([lafa $\underline{\text { a } a ~ P a: x i r a ~ P a n f a: s i h i]) ~}$

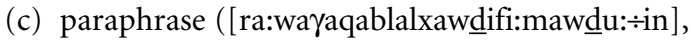
'tergiverser avant d'aborder un sujet')

Les solutions (a) et (c), les plus fréquentes, ne sont pas propres à la traduction interlinguale mais sont en usage chez tout locuteur en situation intralinguale. Malgré la différence fondamentale entre elles, puisqu'en (a) le polylexical est rendu au moyen d'un seul mot, alors qu'en (c), au polylexical figé, on cherche comme équivalent une formation syntagmatique libre, ces deux solutions partagent la particularité de l'abandon du signifiant polylexical de la SF de $\mathrm{L}_{1}$.

Cette rupture fondamentale est-elle inhérente à toute $\mathrm{SF}$ ?

Pour affiner davantage cette question, il faut rappeler l'opposition dénomination directe/dénomination oblique: nous nous servons de ces deux exemples pour illustrer ces deux manières de dénommer: «perdre la vie» et "passer l'arme à gauche». Dans le premier cas, on s'est servi des signifiants disponibles qui entretiennent des rapports de «consubstantialité » sémiotique primaire avec leurs signifiés (qui fait qu'en français 'la vie' est dénommée par «la vie»); dans le second, la consubstantialité des constituants est reléguée au second plan au profit du nouveau signifié (cf. la dualité déjà évoquée). En d'autres termes, les constituants de "passer l'arme à gauche» servent dans leur fonctionnement compositionnel de relais à la dénomination figée, d'où l'oblicité de cette dénomination. Nous avons donc deux types de dénominations qu'on pourrait représenter comme suit: 


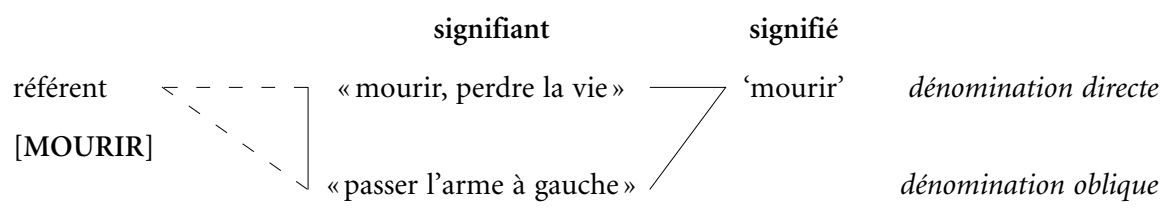

La rupture semble concerner le deuxième type de dénomination. Alors que, dans le premier cas, il est possible d'avoir des correspondants en $\mathrm{L}_{2}$ ([ma:ta] et [faqada haja:tahu]), dans le second, on sacrifie le signifiant imposé par le détour dénominatif.

$\mathrm{Si}$ la désintégration de la matérialité des signes rapproche la poésie et le figement, le traitement des tropes dans les deux cas semble les séparer. Nous avons remarqué que, dans la poésie, la conservation des tropes est assurée lors de la traduction et que cela ne gêne nullement ce transfert d'une langue à une autre. Tel n'est pas le cas des SF: la métaphore à l'origine de «manger les pissenlits par la racine » n'est en aucune façon retenue en $\mathrm{L}_{2}$. Cette différence de traitement des tropes s'explique par le caractère lexicalisé des tropes dans les SF, ce qui les prive de la multiplicité du possible interprétatif des figures vivantes et leur impose, par conséquent, un sens stable d'où la subjectivité de l'interprétant est totalement exclue.

\section{L'intransmissibilité structurelle des JM}

S'il y a un domaine où la traduction joue le rôle d'un repère définitoire, c'est bel et bien celui des JM (Guiraud 1979). En effet, plusieurs auteurs y voient un critère fiable pour distinguer les JM des jeux dits d'esprit (JE):

(a) Dans les JE, tout repose sur l'idée exprimée. Le jeu réside dans la manière dont se présente l'idée. Puisqu'il est de nature onomasiologique, il n'implique pas les signifiants, d'où la possibilité de traduire des jeux comme en (14):

(14) - Quel est le comble de la distraction?

- Avoir perdu ses lunettes et les mettre pour les chercher. (Laclos 1977: 64)

- [ma:hija qimmatussahwi]?

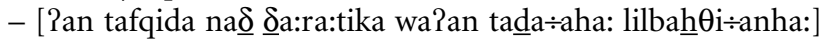

Dans ce genre d'exercice, le jeu est situé clairement au niveau de l'agencement des idées. Dans ce cas précis, il s'agit de chercher des situations qui sont de nature à bien illustrer le terme sur lequel porte la question, ce qu'on peut faire, à la limite, dans n'importe quelle langue.

(b) Dans les JM, la situation est autre: «le point de départ est localisé dans les mots, dans leur matérialité et leur contenu sémantique»; le résultat en est que «le passage d'une langue à une autre nécessite le changement des signifiants [et des signifiés] et, par conséquent, fait perdre au jeu les ingrédients dont il est formé» (Mejri 1997: 7576).

Nous avons eu l'occasion d'opposer JM polylexicaux et unilexicaux (Mejri 1997: 75-92); les premiers héritent des caractéristiques linguistiques que nous avons relevées plus haut. Toute atteinte à ces caractéristiques donne lieu à des manipulations diverses exploitables dans un but ludique: effacement, ajout et substitution de constituants, insertion d'éléments à l'intérieur des SF, détermination d'un constituant à 
l'exclusion des autres, etc. Bref, toute remotivation, de quelque nature qu'elle soit, peut donner lieu à un JM.

Si la SF, surtout à dénomination oblique, résiste à la traduction, que dire du JM auquel elle donne matière? Ici, la rupture lors du passage de $\mathrm{L}_{1}$ à $\mathrm{L}_{2}$ est double: la première concerne la traduction de la SF qui n'a pas nécessairement un correspondant en $\mathrm{L}_{2}$; la seconde donnerait lieu à un énoncé qui ne peut en aucune façon avoir de correspondant en $\mathrm{L}_{2}$ puisqu'il serait en fait la traduction littérale d'un énoncé qui n'aurait rien de ludique. (15) est un exemple où le JM consiste à faire de la SF nominale "petit déjeuner» un verbe capable d'avoir, grâce à ses désinences, toute sorte d'actualisation, jeu facilité par l'origine verbale du second constituant de la séquence nominale:

(15) Il remontait se confectionner du café et petitdéjeunait seul tandis qu'LN dormait encore. (Queneau 1968: 83) [C'est nous qui soulignons.]

La traduction de ce passage en arabe bute nécessairement contre «petitdéjeunait» : d'un côté "petitdéjeuner», à l'origine du JM (le ludé), n'a pas en arabe son correspond littéral [?alfatu:ru issayi:ru] avec l'ordre déterminé + déterminant mais plutôt [fatu:rassaba:hi] 'le déjeuner du matin'. Si, de l'autre, on tente de traduire le JM, on se trouvera dans une situation impossible puisque le correspondant littéral arabe ne renferme pas un élément verbal comme en $\mathrm{L}_{1}{ }^{2}$. Comme on le constate, le JM polylexical initial se désagrège au point de ne trouver, comme c'est le cas dans cet exemple, aucun ancrage linguistique en $\mathrm{L}_{2}$.

Quant au JM unilexical, il implique toutes sortes de jeux sur le signifiant, sur le signifié et sur les emplois syntaxiques ou autres. Or, comme on sait que le signifiant de $\mathrm{L}_{1}$ disparaît purement et simplement pendant la traduction même dans le cas où il y a un correspondant en $\mathrm{L}_{2}$, parce que les mots ne sont pas formés dans les deux langues de la même matière phonique et, par conséquent, n'entretiennent pas avec les autres mots du lexique les mêmes rapports d'homophonie, d'homographie ou de paronymie, que faire par exemple devant un énoncé comme en (16)?

(16) Chaque pas que je fais me rapproche de ce lieu de lucre, de luxe, de luxure huppée où se font et se défont les fortunes des gommeux jeunes et des vieux beaux barons, dissipées - les fortunes - entre les mains des cocodettes et des demi-mondaines à camélias. (Queneau 1968: 65) [C’est nous qui soulignons.]

Les correspondants respectifs arabes de «lucre», «luxe» et «luxure»sont [kasbun], [badaxun] et [daûa:ratun], mots qui ne reprennent évidemment pas la même structure phonique. Il en est de même de «se font» et «se défont» dont les correspondants ne répondent pas à la même configuration morphologique, identité de la base et opposition préfixale; quant à «beaux barons», qui fonctionne ici comme un nom composé (cf. l'emploi du déterminant «des» au lieu de «de»), il nous fournit un bon exemple de manipulations du signifiant: «beaux barons» ne se différencie phoniquement de «beaux-parents» que par l'assourdissement de [b] et l'arrondissement et la postériorité de la nasale.

On peut faire le même raisonnement pour le JM impliquant uniquement le(s) signifié(s) des unités unilexicales. Les deux exemples suivants, tirés du même ouvrage de Queneau, nous éclairent sur l'identité du fonctionnement: 
Docteur

Je vous assure que si vous pouviez me raconter un petit acte manqué, ce serait beaucoup mieux.

Hubert

Je n'écris pas de théâtre.

\section{Docteur}

Je ne comprends pas.

Hubert

Si j'écrivais pour le théâtre, d'abord je n'écrirais pas un seul acte, mais plusieurs; et ensuite, si je n'en écrivais qu'un seul, il ne serait pas manqué.

Docteur

J'entendais dans la vie courante. Ne vous arrive-t-il pas d'oublier vos clefs? de faire des lapsus? de rater un train? de vous tromper d'étage?

(Queneau 1968: 208)

Tout comme le signifiant propre, le signifié n'a d'existence que par rapport à son support phonique: ses emplois dans le discours, les différentes associations qu'il admet et la polysémie qu'il est capable de véhiculer ne peuvent en aucune façon être identiques pour le même mot dans deux langues différentes. C'est la raison pour laquelle les reprises en (17) fondées sur une variation polysémique sont très difficiles à conserver pendant la traduction. Pouvoir les transposer de $\mathrm{L}_{1}$ à $\mathrm{L}_{2}$ présuppose que les mêmes mots dans les deux langues possèdent les mêmes structurations sémantiques, une identité d'emplois discursifs et, par conséquent, les mêmes possibilités de jeux.

En arabe, «acte» a pour correspondants, entre autres, soit [tasarruf] soit [fasll]. Le dernier terme s'emploie aussi bien pour le théâtre que pour les saisons et pourrait donner lieu à des JM qui s'inscrivent nécessairement dans l'organisation sémantique et lexicale de la langue ${ }^{4}$. Faire substituer en (17) à chaque emploi d' "acte» son correspondant arabe détruirait donc tout le JM et, en même temps, toute la trame textuelle créée par ce jeu.

\section{Conclusion}

Partant de ces analyses, nous pouvons dire que la traduction est un exercice révélateur des mécanismes linguistiques qui sous-tendent à la fois les processus mis en place dans chaque système et les usages qu'on en fait. À la lumière des données dégagées des exemples étudiés, il serait possible d'avancer les éléments de conclusion suivants:

1. La traduction est un exercice à valeur heuristique certaine puisqu'il permet d'isoler les spécificités des systèmes linguistiques en place, les zones d'interférence possible, les éléments et les domaines dans chaque système où les distances sont telles que la traduction passe inéluctablement par une forme de réécriture. Cet exercice permet aussi de découvrir le fonctionnement des systèmes dans leur opposition à d'autres systèmes.

2. Cette analyse nous fournit un certain nombre d'éléments qui sont de nature à rapprocher des domaines aussi variés que la poésie, le figement et les JM. Sans la 
traduction, un tel rapprochement n'aurait pas été possible. Parmi les conclusions retenues, nous soulignons cette dimension essentielle, souvent occultée par les analyses, que représente le côté strictement matériel et systémique du langage, c'est-à-dire tout ce qui engage d'une manière ou d'une autre le signifiant et le signifié du signe linguistique, cette dimension étant entendue non pas dans le sens saussurien du terme mais dans celui qui implique toutes les réalisations du signe sur les plans syntagmatique et phrastique (combinaisons phoniques, morphologiques et syntaxiques). Le signifiant renvoie à tout ce qui est trace sonore, que cela couvre les sonorités les plus concrètes et les rythmes les plus récurrents, les structures phoniques ou les combinaisons syllabiques (poésie, JM), ou encore le changement de statut des constituants des séquences (pour le figement). Quant au signifié, il couvre tout ce qui est transfert tropique, sélection sémique ou toutes sortes de manipulations sémantiques (les remotivations, par exemple).

3. Les rapprochements faits entre ces trois domaines grâce à la traduction peuvent servir de point de départ à de nouvelles pratiques didactiques faisant de la traduction non pas un simple exercice de passage de $\mathrm{L}_{1}$ à $\mathrm{L}_{2}$, mais un vrai moyen de découverte des principes de fonctionnement de chaque système linguistique.

4. Parmi les questions théoriques que ces rapprochements éclairent sous un angle nouveau, nous retenons celle de la dissymétrie fondamentale du signe linguistique qui apparaît à travers le fonctionnement des signes dans les domaines étudiés, dissymétrie qui bascule selon les besoins de l'expression du côté du signifiant ou de celui du signifié.

Ainsi s'avère-t-il que l'étude de la traduction ouvre des perspectives énormes devant la recherche en matière de linguistique théorique et appliquée.

\section{NOTES}

1. Le Dictionnaire des expressions et locutions de Rey et Chantreau (1989) mentionne la séquence «prendre une capote» en rapport avec les jeux de cartes.

2. D'autre part, l'arabe ne fait fonctionner qu'exceptionnellement la dérivation à partir de syntagmes.

3. Dans l'exemple (17), le jeu implique à la fois une SF et les unités unilexicales constitutives. Le jeu implique donc les signifiés de chaque mot et le signifié global.

4. Une telle organisation n'est pas impliquée par le jeu d'esprit dont les contours ne dépassent pas l'organisation du discours, et ce, indépendamment du lexique utilisé; c'est pourquoi le même jeu d'esprit peut être rendu ou exprimé différemment sans risque de déperdition majeure. Tant que le jeu ne touche pas au système de la langue (toutes sortes de structuration), il admet normalement la traduction intra- ou inter-linguale.

\section{RÉFÉRENCES}

Apollinaire, G. (1925): Calligrammes, Paris, Gallimard.

Ben Amor, T. (1999): Les jeux de mots chez Queneau, thèse, La Manouba, Faculté des lettres de La Manouba.

Bobin, C.(1992): Le très bas, Paris, Gallimard.

Bonномme, M. (1987): Linguistique de la métonymie, Berne, Peter Lang.

Chuquet, A. et M. Paillard (1989): Approche linguistique des problèmes de traduction, Paris, Ophrys.

Constant, P. (1998): Confidence pour confidence, Paris, Gallimard.

Dancette, J. (1995): Parcours de traduction, Lille, Presses universitaires de Lille. 
Foucault, M. (1988): Les structures linguistiques de la genèse des jeux de mots, Berne, Peter lang. Gross, G. (1996): Les expressions figées en français, Paris, Ophrys.

Gross, M. (1982): «Une classification des phrases «figées»du français», Revue québécoise de linguistique, 11-2.

Guiraud, P. (1979): Les jeux de mots, Paris, Presses universitaires de France, coll. «Que sais-je?», $\mathrm{n}^{\circ} 1956$.

Laclos, M. (1977): Jeux de lettres, jeux d'esprit, Paris, Jean-Claude Simoën.

Ladmiral, J.-R. (1994): Traduire: théorèmes pour la traduction, Paris, Gallimard.

Martin, R. (1992): Pour une logique du sens, $2^{\mathrm{e}}$ éd., Paris, Presses universitaires de France.

MejRi, S. (1997a): Le figement lexical: descriptions linguistiques et structuration sémantique, La Manouba, Publications de la Faculté des lettres de La Manouba.

— (1997b) : «Binarisme, dualité et séquences figées ", Les formes du sens, Mélanges R. Martin, Paris-Gembloux, Duculot.

— (1997c): «Défigement et jeux de mots», Études linguistiques, vol. 3, Tunis, Publications de l'Association Tunisienne de Linguistique.

— (1998a): "Le figement et la linéarité du signe linguistique», L'information grammaticale, numéro spécial «Tunisie», mai 1998.

— (1998b) : «La mémoire des séquences figées: une troisième articulation ou la réhabilitation du culturel dans le linguistique», La mémoire des mots: Actes des VI Journées scientifiques $d u$ Réseau LTT (André CLAS, Salah Mejri et Taïeb Baccouche, dir.), Tunis, AUPELF-UREF, coll. «Actualité scientifique».

- (1999): «Unité lexicale et polylexicalité», Linx, 40.

Petit, G. (1999): «La double hybridation de l'unité lexicale», Linx, 40.

Prandi, M. (1998) : «Les motivations conceptuelles du figement», Le figement lexical: Actes de la $1^{r e}$ RLM (S. Mejri, G. Gross, A. Clas et T. Baccouche, dir.), Tunis, CERES.

Queneau, R. (1968): Le vol d'Icare, Paris, Gallimard.

Radhouane, N. (2000): "Saint-John Perse: le paradoxe de l'hermétisme et de la traductibilité», Meta, 45-3, numéro spécial «La traduction dans le monde arabe».

Rey, A. et S. Chantreau (1989): Dictionnaire des expressions et locutions, Paris, Dictionnaires Le Robert.

Traduction dans le développement des littératures $(\mathrm{La})$ : Actes du XI congrès de l'Association Internationale de littérature comparée (1993), Berne, Peter Lang-Leuven University Press. 